\title{
What Type of Peripheral Loco-Regional Anaesthesia for Thoracic Limb Surgery at the University Hospital La Renaissance - N'Djamena Chad
}

\author{
Kader Ndiaye ${ }^{1, ~ *}$, Sory Traore ${ }^{1}$, Adamou Abbassi ${ }^{1}$, Mbang Dono Djerabe ${ }^{1}$, Adjougoulta Bonte ${ }^{2}$, \\ Kotyade Ngamai ${ }^{3}$, Siniki Fandebnet ${ }^{3}$ \\ ${ }^{1}$ Department of Anaesthesia, Intensive Care at the University Hospital La Renaissance, N'Djaména, Chad \\ ${ }^{2}$ Anaesthesia-Resuscitation Department at the University Hospital La Référence Nationale, N'Djaména, Chad \\ ${ }^{3}$ Department of Orthopaedic and Trauma Surgery at the University Hospital La Renaissance, N'Djaména, Chad
}

Email address:

kader_n@yahoo.fr (K. Ndiaye)

${ }^{*}$ Corresponding author

\section{To cite this article:}

Kader Ndiaye, Sory Traore, Adamou Abbassi, Mbang Dono Djerabe, Adjougoulta Bonte, Kotyade Ngamai, Siniki Fandebnet. What Type of Peripheral Loco-Regional Anaesthesia for Thoracic Limb Surgery at the University Hospital La Renaissance - N'Djamena Chad. International Journal of Clinical and Experimental Medical Sciences. Vol. 7, No. 2, 2021, pp. 50-54. doi: 10.11648/j.ijcems.20210702.13

Received: April 5, 2021; Accepted: April 16, 2021; Published: April 26, 2021

\begin{abstract}
Loco-regional anaesthesia has been revived in recent years, particularly with the advent of ultrasound guidance. We conducted a prospective study over a period of one year on the activity of peripheral loco-regional anaesthesia; 25 patients operated on the thoracic limb who had benefited from peripheral loco-regional anaesthesia were collated. Echo-guidance predominated in $76 \%$ of cases, axillary block predominated in our study, i.e. $72 \%$ of cases; $48 \%$ of our patients had had prior premedication with the combination of hypnovel and low dose fentanyl, which made the nerve block to be carried out more comfortably. The most used products were the combination of Bupivacaine $0.5 \%$ and Lidocaine $2 \%$ in $88 \%$; the average volume injected per plexus block was $21.38 \mathrm{ml}$ with a standard deviation of $6.1 \mathrm{ml}$. The postoperative analgesia was satisfactory with $28 \%$ of the patients having no pain, $64 \%$ having only moderate pain and only $4 \%$ having pain judged to be intense, i.e. 1 patient; the average duration of this postoperative analgesia was 252 minutes, going up to 420 minutes and it is in the same range as that found in the literature, which is $172.8 \mathrm{~min}$ on average, going up to $546.4 \mathrm{~min}$ for certain authors. No side effects or complications were observed in our series. In conclusion, we have noted the advantage of the practice of peripheral loco-regional anaesthesia for both the patient and the hospital; this should encourage practitioners to standardise it.
\end{abstract}

Keywords: Peripheral Loco-Regional Anaesthesia, Thoracic Limb Surgery, Clinical Impact

\section{Introduction}

In recent years, anaesthesia has undergone an enormous evolution, notably the techniques of peripheral loco-regional anaesthesia, which has had a revival, from neurostimulation to ultrasound guidance, and which constitutes a considerable comfort for the patient, especially in terms of reducing the risks associated with general anaesthesia and good management of intra- and post-operative pain.

At the University Hospital La Renaissance, we have made it a priority to use peripheral loco-regional anaesthesia for thoracic limb surgery whenever possible; we conducted a one-year prospective study on the activity of peripheral locoregional anaesthesia for thoracic limb surgery and postoperative follow-up; two techniques were used: ultrasound guidance and neurostimulation. Our objective was to evaluate our practice of peripheral loco-regional anaesthesia and its results in order to propose protocols for the further improvement of our patients' management.

\section{Patients and Methods}

We conducted a prospective study on all patients taken from the operating room for isolated thoracic limb surgery in our University Hospital during the study period, from 01 
January 2020 to 31 December 2020. Only consenting patients who were operated on under peripheral loco-regional anaesthesia, without indication of prior general anaesthesia, were included.

Pre-medication with hypnovel $(2 \mathrm{mg})$ and Fentanyl $(25 \mu \mathrm{g})$ allows reduce pain at the injection site as well as intraoperative anxiety.

The technique used was ultrasound guidance and neurostimulation, sometimes with the coupling of two techniques using echoplex needles $25 \mathrm{~mm}$ to $50 \mathrm{~mm}$, rarely in $100 \mathrm{~mm}$.

The products used were Bupivacaine $0.5 \%$ combined with Lidocaine $2 \%$; this results in concentrations of $0.25 \%$ for Bupivacaine and $1 \%$ for Lidocaine; Ropicavain $0.375 \%$ was rarely used in isolation.

Pain was assessed by visual analogue scale (VAS) at the second, fourth and eighth postoperative hour.

The data were analysed with sphinx Plus 5 software and the graphs with Excel software.

\section{Results}

We collected 25 patients who had received peripheral locoregional anaesthesia out of 31 patients operated on for thoracic limb surgery, i.e. $80.6 \%$ of thoracic limb operations; the average age was 33 years, $84 \%$ adults with extreme ages of 9 and 70 years and a sex ratio $\mathrm{M} / \mathrm{F}=3.1$

For the previous pathological conditions, there was only one case of coronary artery disease and one case of chronic kidney failure.

The injury mechanisms were dominated by assaults (28\%), followed by traffic and road accidents in $32 \%$ of cases (see table 1). And the surgical indications were mainly hand tendinoplasties in $36 \%$ of cases (see table 2 ).

Table 1. Classification according to injury mechanism.

\begin{tabular}{lll}
\hline Mechanism of Trauma 1 & Workforce & Percentage \\
\hline No trauma mechanism & 3 & 12,0 \\
Traffic Accident / MVA & 4 & 16,0 \\
Domestic accident & 4 & 16,0 \\
Assault & 7 & 28,0 \\
Non-traumatic & 6 & 24,0 \\
Panaris / phlegmon & 1 & 4,0 \\
Total & 25 & 100,0 \\
\hline
\end{tabular}

Table 2. Distribution by indication and surgical site.

\begin{tabular}{lll}
\hline Surgical indications & Workforce & Percentage \\
\hline elbow & 2 & 8,0 \\
Debridement of abscesses & 6 & 24,0 \\
Forearm osteosynthesis & 2 & 8,0 \\
Osteosynthesis of the arm & 2 & 8,0 \\
Shoulder osteosynthesis & 1 & 4,0 \\
Hand osteosynthesis & 3 & 12,0 \\
Hand tendinoplasty & 9 & 36,0 \\
Total & 25 & 100,0 \\
\hline
\end{tabular}

Depending on the surgical site and indication, the peripheral loco-regional anaesthesia techniques chosen were predominantly axillary in $72 \%$ of cases, followed by supra- clavicular block in $20 \%$ of cases and $8 \%$ had an additional elbow block (figure 1). The predominant technique used was ultrasound guidance in 19 cases $(76 \%)$, neurostimulation alone in 5 cases $(20 \%)$, and ultrasound guidance coupled with neurostimulation in 1 case (figure 2). $48 \%$ of our patients had been premedicated with hypnovel $2 \mathrm{mg}$ combined with $25 \mu \mathrm{g}$ fentanyl before the nerve block was performed.

The combination of bupivacaine $0.5 \%$ and lidocaine $2 \%$ was used in $88 \%$ of cases and ropivacaine alone in $12 \%$ of cases. The average volume injected per plexus block was $21.38 \mathrm{ml}$ with a standard deviation of $6.1 \mathrm{ml}$.

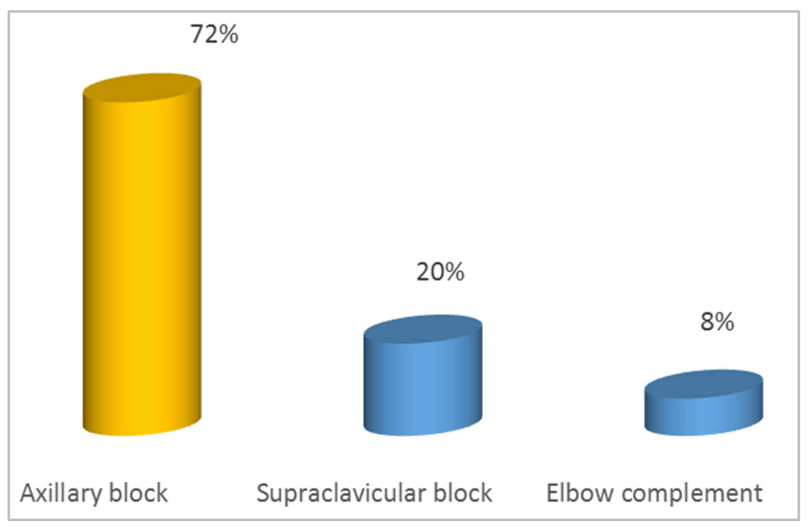

Figure 1. Type of nerve block performed.

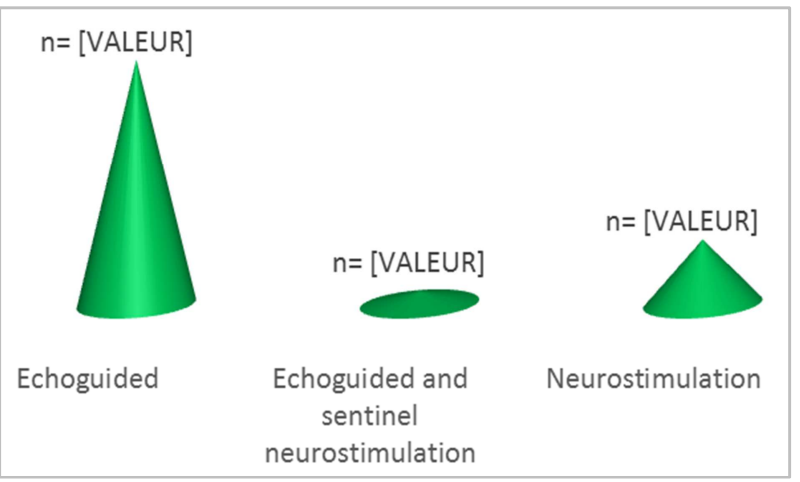

Figure 2. Performing technique of nerve block.

We noted that the sensory and motor blocks were complete in $80 \%$ of cases and in $20 \%$ of cases, the installation of the sensory block was partial.

The average completion time was 7 minutes with a standard deviation of 3 and the average installation time was 8.76 minutes with a standard deviation of 4 .

Postoperative analgesia was assessed at the second hour (H2), fourth hour (H2) and eighth hour postoperative (H8); the VAS (Visual Analogue Scale) was used for pain assessment as described in the following charts:

Table 3. VAS/EVA at H2.

\begin{tabular}{lll}
\hline H2 & Workforce & Percentage \\
\hline$\leq 3$ & 24 & 96,0 \\
4 à 5 & 1 & 4,0 \\
Total & 25 & 100,0 \\
\hline
\end{tabular}


So in table 2 we observe that $96 \%$ of our patients had a VAS $\leq 3$
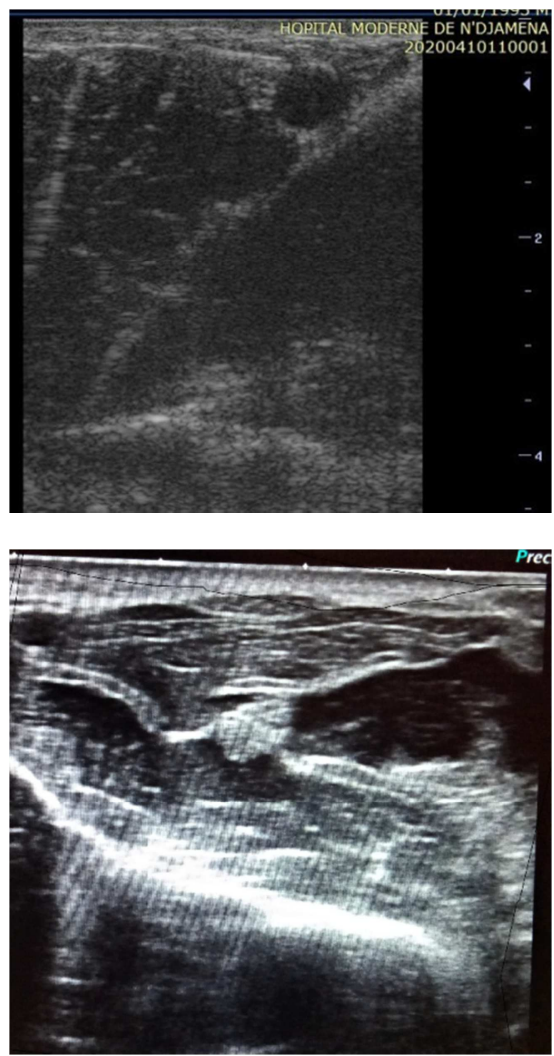

Figure 3. Echo-guided axillary block: Echo-guided median nerve block.

Table 4. VAS/EVA at H4.

\begin{tabular}{lll}
\hline H4 & Workforce & Percentage \\
\hline$\leq 3$ & 18 & 72,0 \\
4 à 5 & 4 & 16,0 \\
Not assessed & 3 & 12,0 \\
Total & 25 & 100,0 \\
\hline
\end{tabular}

At the 4th postoperative hour, the VAS $\leq 3$ was the majority in $72 \%$ of cases and only 3 patients had not benefited from pain assessment, i.e. $12 \%$ of patients.

Table 5. VAS/EVA at H8

\begin{tabular}{lll}
\hline H8 & Workforce & Percentage \\
\hline$\leq 3$ & 7 & 28,0 \\
4 à 5 & 16 & 64,0 \\
6 à 7 & 1 & 4,0 \\
Not assessed & 1 & 4,0 \\
Total & 25 & 100,0 \\
\hline
\end{tabular}

At the 8 th hour, only 1 patient had a VAS between 6 and 7 .

The average duration of the sensory block was 252 minutes and ranged from 120 minutes to 420 minutes, with a standard deviation of 92 . There were no incidents or accidents related to loco-regional anaesthesia.

\section{Discussion}

Fundamental to modern neural blockage is the concept that pain is a sensory warning transmitted by nerve fibres and is subject to modulation or interruption anywhere in the nerve pathway [1, 2]. Peripheral nerve block as an anaesthetic technique plays an important role in loco-regional anaesthesia compared to general anaesthesia and confers excellent pain control and reduction of postoperative stay [2].

In our study, the average age was 33 years with extreme ages of 9 and 70 years and a sex ratio $\mathrm{M} / \mathrm{F}=3.1$ with only $8 \%$ ASA II; our age range is almost similar to other authors age ranging from 19 to 97 years with a sex ratio of 0.6. Unlike our sample, their population was predominantly female [3] and an adult age above 18 years [4].

The mechanisms were dominated by assaults (28\%), followed by traumatic causes such as traffic and road accidents in $32 \%$ of cases (see Table 1); the surgical indications (Table 2) were mainly located in the hand in $48 \%$ of cases.

Several factors and determinants for the choice of the block to be performed - which depend on the lesion location, the history and morphology of the patient - among others, can also influence the choice of the technique. For example, for a morbidly obese patient, an axillary block may be preferred to an infraclavicular block as it is more superficial. Patients with severe lung disease should be carefully evaluated, for phrenic nerve block, a side effect of interscalene and supraclavicular blocks, may precipitate respiratory failure in these patients [5]. The factors determining the nerve territories anaesthetised are a major consideration when choosing a neural block technique [5]; as well as the technical difficulty and/or experience of the practitioner, and finally the benefit/risks ratio of the procedure under consideration [6].

Axillary block predominated in our study, i.e. $72 \%$ of cases, followed by supra-clavicular block in $20 \%$ of cases and $8 \%$ had a complement of elbow block (figure 1) so the initial sensory block was partially installed. The echoguidance technique in $76 \%$, neurostimulation in $20 \%$ and echo-guidance coupled with neurostimulation for 1 case (i.e. $4 \%$ ) (figure 2); in other series echo-guided supraclavicular block and neurostimulation [4] and the failure rate of the block was similar, $8 \%$ in our series vs. $5.1 \%$, up to $12.5 \%$ [4] and a success rate of $94.9 \%$ in their series vs. $76 \%$ in our series. Neurostimulation is still used in our hospital, due to the non-permanent availability of ultrasound in the operating room. Nevertheless, both techniques still exist with a clear trend towards ultrasound guidance; in 2012, in a panel of 247 anaesthetists in France, 16\% of peripheral LRAs were performed using neurostimulation guidance alone. For the remaining peripheral LRAs (84\%), 40\% benefited from ultrasound guidance alone, and $60 \%$ from a combination of both guidance methods neurostimulation and ultrasound guidance [7].

$48 \%$ of our patients had been premedicated with hypnovel $2 \mathrm{mg}$ combined with $25 \mu \mathrm{g}$ fentanyl, compared with $94 \%$ in another study [8]. However, their population was mainly children, unlike our study population, concerning adult; our premedication protocol is similar to the study [9] in terms of the molecules used; other authors had used the same products 
plus adrenaline 1: 200,000 [4].

Adjuvants such as tramadol, dexmedetomidine, magnesium, opioids, adrenaline and naloxone have not been shown to be effective and the benefit-risk balance is unfavourable; the use of adrenaline can be considered for the test dose [10]. The analgesic properties of clonidine as an adjuvant, administered intrathecally or epidurally, have been demonstrated; but the benefit of its addition to local anaesthetics for peripheral nerve blocks is less clear, although it is widely accepted that clonidine improves the quality and duration of a block [11].

Our patients who received premedication did not feel any pain during needle punctures when the block was performed; this low rate of premedication in our sample explains the absence of a standardised protocol in our centre for performing LRA. For premedication, the most used combination is hypnovel between 1 to $2 \mathrm{mg}$ and sufenta $5 \mu \mathrm{g}$ which gives good analgesia during the block [12]. The nonuse of sufenta in our series, whose advantage in loco-regional anaesthesia no longer needs to be demonstrated, is due to the non-permanent availability in our hospital.

The average completion time was $7 \mathrm{~min}$ with a standard deviation of $3.68 \mathrm{~min}$ and $20 \mathrm{~min}$ and the average installation time was 8.76 min with a standard deviation of $4.45 \mathrm{~min}$; in other studies, the average installation time was 13 and 17.5 min [9] and it was $11.57 \mathrm{~min}$ and $21.73 \mathrm{~min}$ respectively by ultrasound and neurostimulation [4] for the same products used separately. Our shorter installation time would be related to the synergy of action of the products.

The use of ultrasound is currently undeniable in the performance of nerve blocks; its advantage is no longer in doubt. In our series, the ultrasound-guided technique was predominant in $76 \%$ of cases; its advantage is irrefutable nowadays as demonstrated in other series. Thus, it also reduces the risk of intravascular injection and damage to the surrounding tissues [3]; the use of ultrasound would have made it possible to have a target zone sparing intra-neuronal injection and other complications such as pneumothorax [13]; but also numerous advantages, in particular the reduction in the time taken to carry out the procedure compared to neurostimulation [4].

The duration of effective analgesia depends on the type of product, the volume and concentration of the local anaesthetic injected, and other patient factors such as diabetic neuropathy, and rarely lasts longer than 16 hours [14]. In our series, postoperative analgesia was satisfactory: $28 \%$ of patients had no pain, $64 \%$ had only moderate pain and only $4 \%$ had pain considered severe, i.e. 1 patient (see chart 5); the average duration of the sensory block was 252 minutes and ranged from 120 minutes to 420 minutes, with a standard deviation of 92 and was 172.8 minutes, going up to 546.4 minutes for some authors [9].

The complications of peripheral loco-regional anaesthesia are numerous: the most frequently cited in the studied literatures are: haematoma, arterial dissection, peripheral neuropathy, pneumothorax, paraesthesia of the hemidiaphragm; accidental intravascular administration leading to a risk of convulsion or ventricular rhythm disorder and risk of cardiac arrest. In relation to the number of procedures, serious complications are rare; identifying the anatomical structures to be avoided theoretically reduces the risk of occurring complications [15]. In our study, we did not note any side effects linked to the technique; other studies have not noted any side effects in their series [4, 8, 9], which reassures and encourages this practice.

\section{Conclusion}

Through this observational study, despite the small size of our sample, the practice of loco-regional anaesthesia has many advantages for both the patients and the practitioners and we were in accordance with several authors. Locoregional anaesthesia provides long-lasting postoperative analgesia. This remains a real comfort for the patients and the technique under ultrasound guidance should be encouraged because its advantages are undeniable and it could avoid many side effects related to the injection. A prospective study with a more exhaustive population in in the future will reinforce this study and this could lead to the establishment of a protocol for loco-regional anaesthesia in our hospital and could encourage other practitioners to use this technique frequently for thoracic limb surgery.

\section{Acknowledgements}

To all the staff of the operating room, the intensive care unit and the surgical department of the University Hospital La Renaissance. My thanks also to Ndiaye Diabe.

\section{References}

[1] David L. Brown B. Raymond Fink. And Michael J Cousins, Phillip O, Bridenbaugh's. The History of Neural Blockade and Pain Management. And Neural blockade in clinical anesthesia and management of pain. $3 \mathrm{r}$ ed. Lippincott-Raven publisher; $1998 ; 3-25$.

[2] Yuvaraj Shashidhar, R. P. Kaushal, Rajkumar Ahirwal. Original Research ArticleA comparative study on upper limb supraclavicular brachial plexus block: dexmedetomidine with ropivacaine and dexamethasone with ropivacaine and ropivacaine alone. International Journal of Health and Clinical Research, 2020; 3 (12S): 14-21.

[3] Robert Almasi, Lajos Bogar, Barbara Rezman, Edina Kovacs, Balazs Patczai, Norbert Wiegand. New composite scale for evaluating peripheral nerve block quality in upper limb orthopaedics surgery. Injury 11 March 2020; 16: 39.

[4] Vinu Mervick Alfred, Gnanasekaran Srinivasan, Mamie Zachariah. Comparison of Ultrasound with Peripheral Nerve Stimulator-guided Technique for Supraclavicular Block in Upper Limb Surgeries: A Randomized Controlled Trial. Anesth Essays Res. 2018 Jan-Mar; 12 (1): 50-54.

[5] Marie-Josee Nadeau, Simon Levesque, Nicolas Dion. Ultrasound-guided regional anesthesia for upper limb surgery. Can J Anesth/J Can Anesth, 2013; 60: 304-320. 
[6] A. Deleuze, M. E. Gentili, F. Bonnet. Description of two techniques of brachial plexus block by infraclavicular approach. Annales Françaises d'Anesthésie et de Réanimation, Feb 2004; 23: 82-84.

[7] Bloc S, Mercadal L, Delbos A, Fuzier R, Narchi P, Ecoffey C. Ultrasound guided regional anesthesia: what is the practice in France in 2012? Abstracts and highlight papers of the 31st annual European Society of Regional Anaesthesia (ESRA) congress 2012. Reg Anesth Pain Med 2012; 37 (7 Suppl): e219.

[8] Markus Zadrazil, Philipp Opfermann, Peter Marhofer, Anna I. Westerlund. Brachial plexus block with ultrasound guidance for upper-limb trauma surgery in children: a retrospective cohort study of 565 cases. British Journal of Anaesthesia; Volume 125, Issue 1, July 2020, Pages 104-109.

[9] Robert Almasi, Barbara Rezman, Zsofia Kriszta, Balazs Patczai, Norbert Wiegand, Lajos Bogar. Onset times and duration of analgesic effect of various concentrations of local anesthetic solutions in standardized volume used for brachial plexus blocks. Heliyon 2020 Sep 2; 6 (9): e04718.

[10] M. Carles, H. Beloeil, S. Bloc, K. Nouette-Gaulain, C. Aveline, J. Cabaton, et al. Perineural loco-regional anaesthesia (PLRA). Formalised expert recommendations Anesth Reanim. 2019; 5: 208-217.
[11] Daniel M. Pöpping, Nadia Elia, Emmanuel Marret, Manuel Wenk, Martin R. Tramèr, David S. Warner, Mark A. Warner. Clonidine as an adjunct to local anaesthetics for peripheral nerve and plexus blocks: A meta-analysis of randomised trials. Anesthesiology August 2009, Vol. 111, 406-415.

[12] Brian P. Kinirons, FFARCSI*, Herve Bouaziz, Xavier Paqueron, Adil Ababou, Celine Jandard, My Maõ Cao, MarieLorraine Bur, Marie-Claire Laxenaire, and Dan Benhamou. Sedation with Sufentanil and Midazolam Decreases Pain in Patients Undergoing Upper Limb Surgery Under Multiple Nerve Block. Anesth Analg 2000; 90: $1118 \pm 21$.

[13] Urooj Siddiqui, Anahi Perlas, Kijinn Chin, Miguel A Reina; Xavier sala-Blanch, ahtsham Niazi and Vincent Chan. Intertruncal approach to the supraclavicular brachial plexus, current controversies and technical update: a daring discourse Regional Anesthesia \& Pain Medicine. 11 March 2020; 45 (5): 377-380.

[14] E. Albrecht and K. J. Chin. Advances in regional anaesthesia and acute pain management: a narrative review. Anaesthesia 2020, 75 (Suppl. 1), e101-e110.

[15] High Authority for Health. Ultrasound guidance for peripheral loco-regional anaesthesia. March 2014: P7-8. 